\title{
Cambios en el rendimiento del alumno universitario de la docencia tradicional a la docencia remota de emergencia Changes in the performance of university students from traditional teaching to emergency remote teaching
}

\author{
Miguel Ángel Montañés Del Río, Vanessa Rodríguez Cornejo, Margarita Ruiz Rodríguez \\ miguelangel.montanes@uca.es: vanesa.rodriguez@uca.es; margarita.ruiz@uca.es \\ ${ }^{1}$ Organización de Empresas \\ Universidad de Cádiz \\ Jerez de la Frontera (Cádiz), España
}

\begin{abstract}
Resumen- El objetivo de este trabajo es poner de manifiesto el proceso de adaptación, como una consecuencia obligada por la COVID-19, de la docencia presencial a la docencia online en una asignatura del Grado en Administración y Dirección de Empresas de la Universidad de Cádiz (en lo sucesivo UCA). Así, se comparará el rendimiento del alumnado con este nuevo modelo de docencia, con el que se había alcanzado en el modelo de docencia tradicional. Para ello, se analizarán las herramientas empleadas tanto para el desarrollo de las clases de manera online a través del Campus Virtual, como para la evaluación de la asignatura. Y se compararan los rendimientos obtenidas por el alumnado matriculado en el curso 2018/2019, impartido mediante docencia tradicional, y el matriculado durante el curso 2019/2020
\end{abstract}

Palabras clave: Docencia Remota de Emergencia, COVID-19, Rendimiento Académico

Abstract- The objective of this work is to highlight the adaptation process, as a consequence forced by COVID-19, from face-to-face teaching to online teaching in a subject of the Degree in Business Administration and Management at the University of Cádiz (in hereinafter UCA). Thus, the performance of the students will be compared with this new teaching model, with the one that had been achieved in the traditional teaching model. For this, the tools used both for the development of the classes online through the Virtual Campus, and for the evaluation of the subject will be analyzed. And the returns obtained by the students enrolled in the 2018/2019 academic year, taught through traditional teaching, and those enrolled during the 2019/2020 academic year will be compared.

Keywords: Remote Emergency, COVID-19, Academic Performance

\section{INTRODUCCIÓN}

La exigencia de la situación generada respecto al teletrabajo por la pandemia de la COVID-19 a partir del 14 de marzo de 2020 en España, ha puesto de manifiesto la necesidad de unas competencias digitales, por parte del profesorado, que hasta ahora, y en muchos casos, se adquirían mediante la formación voluntaria, o de manera autodidacta. Unas competencias que van de la mano de la actual necesidad de facilitar al alumnado algo más que los recursos que hasta ahora se les ha ido suministrando.
La educación no presencial o virtual permite ofrecer una solución a la suspensión de las clases presenciales universitarias, decretada a raíz del problema de salud pública generado por la COVID-19, pues hace posible la enseñanza y el aprendizaje en cualquier lugar y momento. No obstante, este cambio se ha producido a una velocidad asombrosa y sin precedentes (Hodges, Moore, Lockee, Trust, \& Bond, 2020).

Los campus virtuales, que antes eran una herramienta de apoyo para la docencia, pasaron entonces a ser la herramienta clave para poder desarrollar las tareas docentes.

En España, a pesar de que el Personal Docente e Investigador (PDI) de las universidades hacen uso de las TIC's en su día a día, según el Informe del Ministerio de Universidades y utilizando datos referidos al Sistema Universitario Español del curso 2018-2019, el 90.55\%de aquél usa el campus virtual o la docencia virtual poniéndose de manifiesto que las universidades españolas cuentan con tecnología avanzada para impartir docencia online.

Sin embargo, no fue sino hasta la llegada de la pandemia de la COVID-19 cuando se dio una situación en la que se tenían que aunar tres factores: 1) la docencia online impartida en las universidades públicas españolas; 2) un PDI que de forma innata carecía de los conocimientos tecnológicos para llevarla a cabo, y; 3) la inmediatez de poner en práctica una docencia no presencial (Torecillas, 2020).

Este abrupto cambio en la docencia ha dado lugar a una "enseñanza remota de emergencia" (Hodges et al., 2020), pues lo inmediato del cambio hace que esta modalidad de educación no cumpla con el concepto de educación no presencial, conocido hasta ahora y bien discutido por la literatura (Means et al., 2014), pues no deja tiempo para la planificación y el diseño de la experiencia docente.

Mientras que la eduación a distancia no presencial trata de responder a las necesidades de aprendizaje mediante prácticas educativas abiertas (Bozkurt, 2019a; 2019b; Zawacki-Richter et al., 2020), caracterizadas por la distancia en el tiempo y en el espacio entre el alumnado y los recursos necesarios para el desarrollo de su aprendizaje; la enseñanza remota es una solución temporal a un problema puntual (Golden, 2020), que 
supone solo una distancia espacial pero no temporal, permitiendo un aprendizaje sincrónico.

Además, se podría observar otra diferencia entre ambos tipos de educación, como el hecho de que la enseñanza remota a distancia es una obligación, mientras que la enseñanza no presencial u online es una opción (Bozkurt y Sharma, 2020).

Para que la enseñanza remota de emergencia tenga éxito deben superarse tres aspectos fundamentales: 1) la Implementación de recursos tecnológicos, 2) la capacitación del personal docente, y; 3) la adaptación del alumnado a esta forma de educación. Todo ello, sin olvidar que cada uno de estos aspectos ha de enfocarse hacia la calidad educativa.

Por lo tanto, para garantizar la calidad del proceso educativo sin presencialidad física habría de buscarse y perfeccionarse la utilización de las TIC'S que permitan un aprendizaje sincrónico.

\section{A. Tecnologías de la información y de la comunicación} y aprendizaje sincrónico

Aplicada a la docencia, las TIC's persiguen apoyar, mejorar y optimizar la distribución de la información para aumentar el aprendizaje del alumnado, pudiendo considerarse como un subcampo de la Tecnología Educacional (Jamkar \& Shelke, 2020).

Las nuevas tecnologías permitirían al profesorado mayores oportunidades para involucrar al alumnado en su proceso de aprendizaje (Carlson, 2011).

Entre las condiciones necesarias para implementar las TIC's están el hecho de que el profesorado debe no solo tener los conocimientos y habilidades necesarios para usar las nuevas herramientas y recursos digitales, sino también las competencias relacionadas con los contenidos, pedagogía, colaboración y redes que les haga poder utilizarlos activamente en su práctica diaria (Khan, 2020).

Las TIC's permiten el aprendizaje sincrónico, es decir, la utilización tanto por docentes como por discentes, de herramientas multimedia para interactuar en vivo dentro de entornos digitales (Phelps \& Vlachopoulos, 2020). Las herramientas de comunicación sincrónicas permiten espacios virtuales de comunicación en tiempo real en los que docentes y discentes pueden intercambiar información a través de videoconferencias, chat, pizarra virtual, etc. (Grimaldo et al., 2014).

El aprendizaje sincrónico facilita al alumnado su participación en actividades colaborativas, discusiones, lecciones, etc., que son realizadas dentro de una clase virtual (Bower et al., 2014 citado en Phelps \& Vlachopoulos, 2020). Pero tiene algunas limitaciones (Phelps \& Vlachopoulos, 2020): la habilidad del profesorado para su manejo (ser competentes a la hora de manipular y gestionar las herramientas del entorno sincrónico: cámara web, micrófonos, teclados inteligentes, etc.), la gestión de la comunicación, o el diseño de actividades para este entorno.

Aun no sabiendo qué herramienta tecnológica escoger de entre las posibles alternativas, sí se tenía claro que la videoconferencia o el webinar había de ser el método de enseñanza-aprendizaje más adecuado a las actuales circunstancias.
Las videoconferencias suponen la transmisión de texto, gráficos, audio y/o contenidos visuales a través de Internet, lo que requiere el uso de un ordenador y un explorador web, permitiendo que la comunicación pueda ser tanto sincrónica como asincrónica (Khan, 2020).

En la experiencia de teledocencia llevada a cabo en la Universidad de Cádiz, como se verá más adelante, se utilizaron tres herramientas alternativas de videoconferencia: BigBlueButton, Adobe Connect y Google Meet.

\section{Bigbluebutton}

BigBlueButton es un sistema de conferencias web de código abierto para el aprendizaje en línea, lo que significa que: 1) mediante una licencia se puede tener acceso total al código fuente para poder mejorar la aplicación; 2) al tratarse de un sistema de conferencia web, se obtienen las mismas funcionalidades que un sistema comercial, es decir, compartir en tiempo real audio, vídeo, presentaciones y pantalla, además de otras herramientas como la pizarra, las notas compartidas, las encuestas, o la grabación de las sesiones para su posterior visionado; 3) al tener como objetivo el aprendizaje en línea, es un sistema integrado en la mayoría de los principales sistemas de gestión de aprendizaje o LMS (Learning Management Systems) (BigBlueButton Inc., 2020).

\section{Adobe Connect}

Una reunión mediante Adobe Connect es una conferencia online y en directo entre varios usuarios. La sala de reuniones es una aplicación que contiene diversos paneles de visualización, o pods, con funciones y componentes específicos (cámara web, opciones de audio, chat, etc.). De este modo, es posible seleccionar entre varios diseños predeterminados de salas de reuniones, o crear un diseño personalizado adaptado a las necesidades del orador. En la sala de reuniones los asistentes pueden compartir la pantalla, archivos de su equipo, chatear, transmitir audio y vídeo, o participar en actividades en línea interactivas (Adobe Systems Inc., 2020). Ello permitiría, por ejemplo, abrir diapositivas en PowerPoint o mostrar multitud de sitios web (Carlson, 2011).

\section{Google Meet}

Google ha realizado movimientos hacia el nicho de mercado del video chat durante la pandemia de la COVID-19, lo que supone en la práctica competir directamente con otros servicios como Zoom. Ahora ofrece su servicio Premium de videoconferencias, Google Meet, totalmente gratis para los usuarios, ya que anteriormente solo era posible siempre que la organización de pertenencia utilizase G Suite (Rayome, 2020). Se incluirá el número de subsecciones necesarias en cada sección.

El objetivo de este trabajo es poner de manifiesto el proceso de adaptación, como una consecuencia obligada por la COVID19, de la docencia presencial a la docencia online en una asignatura del Grado en Administración y Dirección de Empresas de la Universidad de Cádiz (en lo sucesivo UCA). Así, se comparará el rendimiento del alumnado con este nuevo modelo de docencia, con el que se había alcanzado en el modelo de docencia tradicional.

\section{Contexto}


Dirección de Operaciones I es una asignatura cursada de forma obligatoria en el tercer curso del Grado en Administración y Dirección de Empresas, y en el quinto curso del Doble Grado en Derecho y Administración de Empresas, ambos impartidos por la Universidad de Cádiz. Incorpora 4 ECTS (European Credit Transfer and Accumulation System) teóricos y 2 ECTS prácticos distribuidos a lo largo de tres grupos debido a la elevada matriculación del alumnado en la misma.

Las clases presenciales teóricas se impartían siguiendo la técnica de la lección magistral, mientras que las clases prácticas se desarrollaban a través de la resolución de casos prácticos relacionados con los temas impartidos.

\section{DESCRIPCIÓN}

Para alcanzar los objetivos propuestos, en primer lugar, se discutirá el proceso de adaptación de la asignatura y las herramientas empleadas para que se produjese la misma. En segundo lugar, se observará la percepción del alumnado con la docencia implantada partiendo del estudio de los datos obtenidos en un cuestionario, y en tercer y último lugar, se compararán los resultados obtenidos por el alumnado en la asignatura durante en el curso 2018/2019 con los obtenidos en el curso 2019/2020. Se pretende con ello confirmar, o no, si se ha producido incremento o disminución del rendimiento tras los cambios introducidos. Debe contener las actividades del trabajo y recursos utilizados: es decir, metodologías, técnicas y tecnología.

\section{Resultados}

La primera clase de Dirección de Operaciones I (en adelante DOPI) tras la declaración del estado de alarma en España, se produjo el 16 de marzo. En ella tenía que reanudarse la explicación, de manera online, del tema 4 del programa de la asignatura que ya se había comenzado el último día previo a la suspensión de la docencia presencial.

El primer problema que surgió fue el de elegir la herramienta a utilizar. Se optó inicialmente por BigBlueButton, una aplicación para celebrar reuniones virtuales a través de salas en las que el presentador (en principio, aunque no necesariamente, el docente) puede compartir archivos y realizar grabaciones de lo que ocurre en ellas (Universidad de Cádiz, n.d.), como ya se ha expuesto, que además está disponible dentro del Campus Virtual de la UCA y que cuenta con tutoriales a través de un curso en abierto impartido por esta institución. En el caso de la asignatura analizada, esta herramienta nos permitía compartir pantallas con los alumnos para presentar las diapositivas, emplear el chat para comunicarnos con aquellos alumnos que no tenían la posibilidad de encender el micrófono y grabar la sección

En el caso de la UCA, esta herramienta de videoconferencia se halla integrado dentro de la plataforma Moodle, utilizada para soportar el aula virtual de las asignaturas de la Institución. Ello implica que el usuario no ha de realizar ninguna otra acción más que acceder al enlace de la reunión con BBB situado en el aula virtual del curso correspondiente.

El problema que planteó la utilización de BBB fue que al tratarse del medio más directo con el que contaba el profesorado, su uso masivo, pero, y, sobre todo, el abuso de una de sus aplicaciones (la grabación de las sesiones), se llegase a consumir un volumen considerable de datos del Campus Virtual. Ello derivó en un comunicado que desaconsejaba las grabaciones indiscriminadas.

Partiendo de la idea subjetiva de que la grabación de las sesiones es una de las mayores ventajas de las videoconferencias, la limitación expuesta suponía impedir atender las clases, en un momento posterior, bien al alumnado que hubiera tenido problemas de conexión, bien al que no hubiera podido llegar a tiempo a la reunión o que simplemente le hubiera sido imposible estar virtualmente presente.

Por todo lo anterior, y debido a que los servidores empezaron a resentirse por la gran cantidad de datos que eran grabados en las sesiones de BigBlueButton celebradas por el profesorado de otras muchas asignaturas, se decidió abandonar esta herramienta y utilizar Adobe Connect.

Adobet Connect es una multiplataforma para realizar igualmente reuniones virtuales, o conferencias web sincrónicas, disponible tanto para colegios como para universidades (Cappiccie \& Desrosiers, 2011). Tan solo necesita de un navegador web, y no requiere la instalación previa de aplicaciones cliente (Grau-Moracho, 2007).

El Área de Tecnologías de la Información de la UCA facilitó una cuenta de anfitrión para cada titulación, de forma que sus coordinadores creaban y habilitaban tantas salas como fueran necesarias según la organización de cada asignatura. Las sesiones también podían ser grabadas, solo que esta vez en servidores ajenos a la UCA, y posteriormente ser descargadas por el usuario con lo que, en principio, el problema de la capacidad de almacenamiento estaba solventado. Además permitía como la anterior compartir la pantalla con los alumnos y emplear el chat para comunicarnos con ellos.

En su aplicación a la experiencia de teledocencia aquí expuesta, el principal problema que se observó fue la limitación en el número de asistentes a la reunión. De este modo, cuando se llegaba a un total de cien de ellos (docente incluido), el sistema denegaba el acceso y no permitía la entrada de más asistentes. La asignatura Dirección de Operaciones I contaba, solo para el Campus de Jerez de la Frontera, con un total de 140 personas matriculadas. En el momento de mayor audiencia registrado en una de sus sesiones, la asistencia llegó hasta las 118 personas.

El problema anterior se agravaba cuando quien sí pudo atender a tiempo la entrada en la reunión, por circunstancias ajenas a su voluntad perdía momentáneamente la conexión a Internet. Su "puesto" en el aula virtual podía ser ocupado por otra persona que hasta entonces hubiera estado esperando pacientemente a que el sistema le permitiera el acceso a la sala de reuniones en detrimento, ahora, del alumnado al que le falló la conexión. Éste quedaba fuera de la sala y pasaba a la lista de espera.

Este fue el motivo por el que, a pesar de poder contar con la funcionalidad de la grabación, se decidió emigrar hacia otra herramienta de videoconferencia.

En marzo de 2020, y como respuesta a la crisis de la COVID19, Google comenzó a ofrecer las funciones avanzadas de su herramienta Meet, que anteriormente requerían de una cuenta de empresa, a cualquier usuario que utilizara G Suite o G Suite for Education (Soltero, 2020). Ante ello, y las quejas previas del alumnado por el uso de Adobe Connect, se optó por impartir 
el resto de las clases teóricas de la asignatura a través de Google Meet, un servicio gratuito que permite la realización de videoconferencias y también su grabación, compartir pantalla y comunicarnos por chat.

Por último, será objeto de análisis la diferencia en el rendimiento del alumnado tras el paso desde una docencia en formato tradicional, a una docencia remota de emergencia.

El sistema de evaluación de la asignatura DOPI, cuando es impartida de forma presencial, consiste en un examen de 20 preguntas tipo test sobre los contenidos teóricos de la misma, de forma que cada respuesta correcta es valorada con 0.5 puntos y cada respuesta errónea resta un $30 \%$. Tiene una ponderación de 4 puntos sobre la nota final de la asignatura, y se ofrece un tiempo máximo para su contestación de 1 hora. También consta de un examen práctico con tres ejercicios a resolver mediante el planteamiento y desarrollo de los mismos, para lo que se ofrece un tiempo de ejecución de 1 hora y 15 minutos. Los dos primeros ejercicios tienen suelen tener una valoración de 1,5 puntos y el tercero de ellos de 1 punto. Se impone, además, el requisito de obtener un $30 \%$ de la nota de cada uno de estos ejercicios prácticos para poder sumar la nota del examen práctico a la nota del examen teórico.

Sin embargo, el sistema de evaluación durante el periodo que se impartió la asignatura en modalidad online mediante el campus virtual de la misma consistió en un examen tipo test de 20 preguntas correspondientes a la parte teórica de la misma. Cada respuesta correcta sumaba 0.5 puntos, y cada una de las incorrectas restaba un 30\%. Suponía una nota máxima de 4 puntos sobre la nota final, y debía realizarse en un tiempo de ejecución de 1 hora. También hubo un examen práctico con tres ejercicios, con una apuntación máxima de 4 puntos sobre la nota total de la asignatura, pero cuya ejecución y desarrollo no fueron necesarios entregar. Antes bien, cada ejercicio práctico se acompañaba de varias preguntas tipo test (de 2 a 5 dependiendo del ejercicio), y se dispuso de un tiempo de realización máximo de 1 hora y 15 minutos. Los dos primeros ejercicios tuvieron una valoración de 1,5 puntos mientras que el tercero de ellos se ponderó con 1 punto. Se mantuvo, de igual forma, el requisito de obtener al menos un $30 \%$ de la nota de cada uno de los ejercicios prácticos propuestos para poder sumar, a la nota del examen práctico, la nota obtenida en el examen teórico.

Para poder comparar la diferencia en cuanto a los resultados obtenidos entre ambos periodos estudiados, en primer lugar, se estudian las notas obtenidas por el alumnado en la convocatoria ordinaria de junio y extraordinaria de septiembre de los cursos $2018 / 2019$ y $2019 / 2020$.

En la figura 1 se aprecian los porcentajes asociados a las correspondientes calificaciones finales obtenidas por el alumnado durante la convocatoria ordinaria del mes de junio del curso 2018/2019. Para la misma, había un total de 90 personas matriculados en la asignatura.

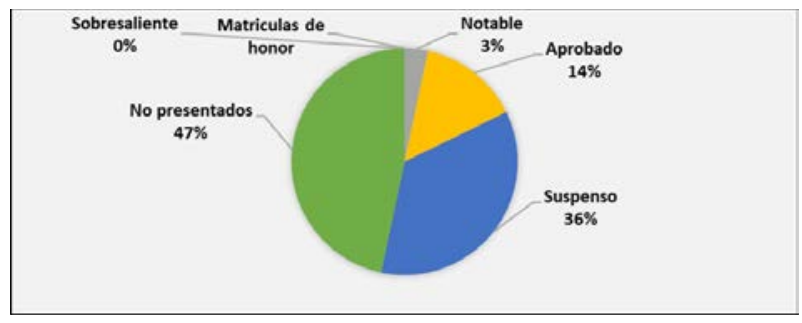

Figura 1. Calificaciones obtenidas por el alumnado matriculado en DOPI en la convocatoria de examen de junio de 2019 de DOPI

Como se observa en la figura 1, la asignatura DOPI tuvo en la convocatoria de examen de junio de 2019 un alto índice de alumnado no presentado (47\%), así como también un alto índice de alumnado suspenso (36\%). Al respecto del alumnado que consiguió superar la asignatura, un $14 \%$ y un 3\% del mismo obtuvo, respectivamente, una calificación de aprobado y de notable. Nadie alcanzó, sin embargo, la calificación de sobresaliente o de matrícula de honor.

En la convocatoria de examen de septiembre de 2019 (véase la figura 2), por su parte, tanto el porcentaje de alumnado no presentado como el del alumnado suspenso, siguen siendo muy altos. Un $46 \%$ y un $38 \%$, respectivamente. Respecto al alumnado que superó la asignatura, un $8 \%$ del mismo consiguió un aprobado, y otro $8 \%$ alcanzó una calificación de notable.

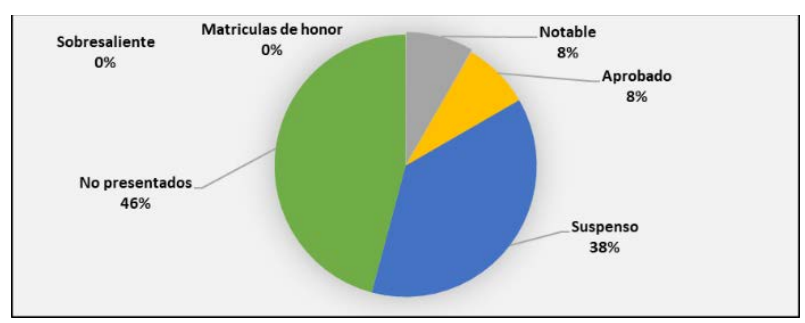

Figura 2. Calificaciones del alumnado en la convocatoria de examen de septiembre de 2019 en DOPI

En la figura 3 se pueden observar los porcentajes con las correspondientes a las calificaciones finales obtenidas en la convocatoria ordinaria de examen de junio del curso 2019/2020 por el alumnado matriculado en DOPI (un total de 108 estudiantes).

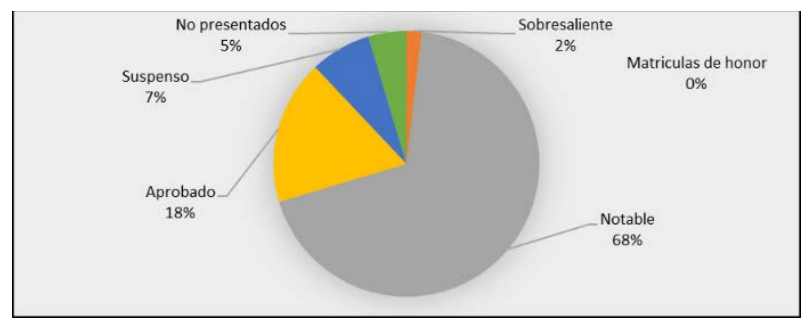

Figura 3. Calificaciones del alumnado en la convocatoria de examen de junio de 2020 en DOPI

En la convocatoria de examen de junio de 2020, y con un examen totalmente online, el porcentaje del alumnado que dejó de presentarse fue bajo en relación a la misma convocatoria del año anterior $(5 \%)$. De igual modo, el porcentaje de suspensos no alcanzó las dos cifras (7\%). De entre quienes consiguieron superar la asignatura (88\%), un 18\% alcanzó la calificación de aprobado, un $68 \%$ la de notable, y tan solo un $2 \%$ consiguió llegar al sobresaliente. 
En la convocatoria de examen de septiembre de 2020 (figura 4), el porcentaje del alumnado no presentado fue del $31 \%$, llegando al 7\% la cifra de quienes suspendieron. Por su parte, un $31 \%$ del alumnado llegó a la nota de aprobado, y otro $31 \%$ a la de notable.

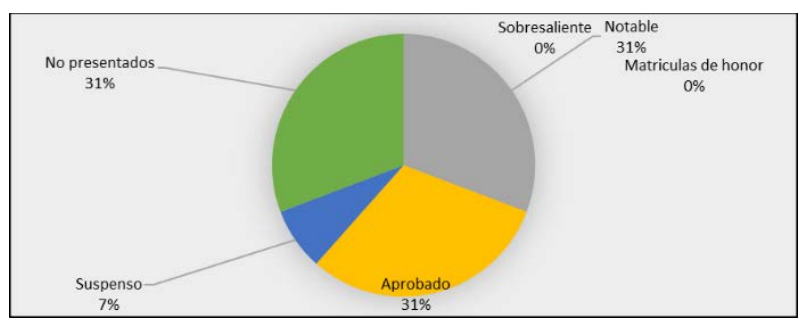

Figura 4. Calificaciones del alumnado en la convocatoria de examen de septiembre de 2020 en DOPI

Es fácilmente comprobable el hecho de que el número de estudiantes presentados a la convocatoria de examen de septiembre disminuyó considerablemente, mientras que el número de estudiantes aprobados se incrementó del curso 2018/2019 al curso 2019/2020.

\section{CONCLUSIONES}

La interrupción de la docencia presencial debida a la crisis sanitaria generada por la pandemia de la COVID-19 ha provocado que se resientan los cimientos de esta modalidad educativa, tal y como se la conoce hoy día, al menos a nivel universitario.

Ha surgido la necesidad de buscar soluciones de manera rápida y sin planificación ni preparación alguna por parte del profesorado y del alumnado. La aplicación de la enseñanza remota de emergencia, como solución puntual a un problema muy concreto, y aun cuando la misma no deba de entenderse como un tipo de docencia que haya de mantenerse en el tiempo, sí que sería necesario incorporar parte de ella en la preparación de futuros cursos ante la previsión de nuevas situaciones de crisis mundial. Ello quizás permitiría una docencia sin cambios abruptos e inmediatos.

El objetivo de este artículo era analizar el proceso de adaptación de la docencia presencial a la docencia remota de emergencia de una asignatura del Grado en ADE, Dirección de Operaciones I, así como exponer las características de las herramientas empleadas para hacer frente a esta nueva situación, mostrando sus pros y sus contras. Ello debería haber podido ayudar al profesorado a tomar decisiones acerca de cuál de ellas utilizar en cada momento. También se ha estudiado cómo han evolucionado las calificaciones del alumnado desde la docencia presencial hasta la docencia online.

Una vez consultada la literatura, se podría concluir que las videoconferencias serían una de las herramientas que mayores ventajas presentan a la hora de poder continuar con una docencia no presencial pero sincrónica pues, de este modo, la interacción docente-discente se sigue manteniendo como en una situación "normal".

Si bien las herramientas elegidas para ello, en un principio BigBlueButton y Adobe Connect, ya habían sido incorporadas por la UCA a su Campus Virtual, habían sido hasta la llegada de la COVID-19 poco empleadas para la docencia presencial, y finalmente presentaron importantes deficiencias permitieron una calidad adecuada en la docencia a distancia.
Aun cuando BigBlueButton no limitaba el número de discentes que podían conectarse a una sesión, no soportó el elevado número de conexiones simultaneas derivado de su uso por la práctica totalidad del profesorado de todas las asignaturas de los diferentes Grados impartidos en la UCA. Ello impidió, por ejemplo, la grabación de las sesiones, acción fundamental para que el alumnado con dificultades de conectividad tuviera la posibilidad de rescatar la clase en un momento posterior del tiempo, y no perderse así contenidos.

Adobe Connect no presentó problemas de conexión y permitió no solo compartir recursos con el alumnado, sino también que las sesiones se grabaran sin presentar complicaciones. Sin embargo, limitaba la asistencia por sesión a un total de 100 personas incluido el profesorado. En asignaturas con un alto número de matriculados, como lo es Dirección de Operaciones I, fue un gran inconveniente que provocó la frustración y la desmotivación del alumno, quien no solo tenía que estar preparado para entrar en la sesión minutos antes de que esta comenzara, y así esperar ser de los 100 primeros en hacerlo, sino también no perder de vista la cola de espera para volver a entrar en el caso de que la conexión le fallara temporalmente y fuera expulsado de la videoconferencia.

Para apaliar las limitaciones de las anteriores herramientas se empleó Google Meet, que a pesar de ser de reciente creación, y por lo tanto presentar menor experiencia entre el profesorado y el alumnado, fue la que menos problemas presentó.

La docencia no presencial, sobre todo si se quiere seguir manteniendo la interconexión con el alumnado, requiere de un tiempo para su preparación y su posterior adaptación, pero también, $\mathrm{y}$, sobre todo, precisa de un rediseño de las herramientas y tecnologías empleadas para ello. No se puede pretender replicar en un entorno virtual o a distancia la docencia tal y como se conocía hasta ahora, lo que implica que el profesorado ha de tener capacidades tecnológicas que le permitan conocer las posibilidades que ofrecen las distintas herramientas de las que se dispone. Pero también sus limitaciones, no solo a la hora de impartir una docencia de calidad, sino también en el momento de presentarla a un público objetivo muy concreto, pues, aunque la pandemia de la COVID19 haya cambiado todo el proceso de enseñanza-aprendizaje, ello no debe suponer una apatía en el alumnado fruto de la desmotivación y el sentimiento de abandono por parte de su profesorado.

En lo relativo al modelo de evaluación, al igual que la docencia hubo que cambiarlo y ajustarlo a la nueva forma de impartila. El examen presencial dio paso a los exámenes online en forma de tipo test no solo para la teoría sino también para la práctica.

Respecto al análisis de los resultados podemos observar que el número de alumnos nos presentados a la asignatura de un curso a otro disminuye significativamente, sin embargo, el número de alumnos que superan la asignatura con éxito no solo se incrementa, sino que se elevan las calificaciones pasando a tener un mayor número de notables.

\section{REFERENCIAS}

Adobe Systems Inc. (2020). Guía del usuario de Adobe Connect. Retrieved June 18, 2020, from https:/helpx.adobe.com/es/adobe-connect/using/userguide.html 
BigBlueButton Inc. (2020). BigBlueButton Documentation. Retrieved June 20, 2020, from https://docs.bigbluebutton.org/

Bower, M., Dalgarno, B., Kennedy, G., Lee, M. J. W., \& Kenney, J. (2014). Blended Synchronous Learning-A Handbook for Educators Blended synchronous learning: a handbook for educators 2. http://blendsync.org

Bozkurt, A. (2019b). Intellectual roots of distance education: a progressive knowledge domain analysis. Distance Education, 40(4), 497-514.

Bozkurt, A., \& Sharma, R. C. (2020). Emergency remote teaching in a time of global crisis due to CoronaVirus pandemic. Asian Journal of Distance Education, 15(1), 2020. https://doi.org/10.5281/zenodo.3778083

Golden, C. (2020). Remote teaching: The glass half-full. Educause Review.

Grau-Moracho, J., \& Jordi Grau-Moracho, P. (n.d.). Title: Virtual meetings: Adobe Connect and WebEx Reuniones virtuales: Adobe Connect y WebEx. https://doi.org/10.3145/epi.2007.sep.16

Hodges, C., Moore, S., Lockee, B., Trust, T., \& Bond, A. (2020a). The Difference Between Emergency Remote Teaching and Online Learning. Educause Review, 7.

Hodges, C., Moore, S., Lockee, B., Trust, T., \& Bond, A. (2020b). The Difference Between Emergency Remote Teaching and Online Learning. In medicine.hofstra.edu. https://er.educause.edu/articles/2020/3/the-differencebetween-emergency-remote-teaching-and-

Khan, A. (2020). Information Communication Technology in Higher Education. In Ideal Research Review (Vol. 1, Issue 21).
Education and Training, ITHET https://doi.org/10.1109/ITHET.2012.6246041

2012.

Ladson-Billings, G. J. (1999). Preparing teachers for diverse student populations: A critical race theory perspective. Review of Research in Education, 24, 211-247. https://doi.org/10.3102/0091732x024001211

Means, B., Bakia, M., \& Murphy, R. (2014). Learning online: What research tells us about whether, when and how. Routledge.

Moodle, C. (2007). Acerca de Moodle.

Phelps, A., \& Vlachopoulos, D. (2020). Successful transition to synchronous learning environments in distance education: A research on entry-level synchronous facilitator competencies. Education and Information Technologies, 25(3), 1511-1527. https://doi.org/10.1007/s10639-01909989-x

Wang, S., Minku, L. L., \& Yao, X. (2018). A Systematic Study of Online Class Imbalance Learning with Concept Drift. IEEE Transactions on Neural Networks and Learning Systems, 29(10), 4802-4821. https://doi.org/10.1109/TNNLS.2017.2771290

Zawacki-Richter, O., Conrad, D., Bozkurt, A., Aydin, C., Bedenlier, S., Jung, I., Stöter, J., Veletsianos, G. M., Blaschke, L., Bond, M., Broens, A., Bruhn, E., Dolch, C., Kalz, M., Kondakci, Y., Marin, V., Mayrberger, K., Müskens, W., Naidu, S., ... Kerres, M. (2020). Elements of Open Education: An Invitation to Future Research. International Review of Research in Open and Distributed Learning, 21(3), 319-334. https://doi.org/10.19173/irrodl.v21i3.4659 